\title{
Magnetic field of the Sun as a star and two solar-like stars $\xi$ Boo $\mathrm{A}$ and $61 \mathrm{Cyg} \mathrm{A}$.
}

\author{
Sergei I. Plachinda ${ }^{1}$ \\ ${ }^{1}$ CAO, Nauchny, Crimea, 98409 Ukraine; email: plach@crao.crimea.ua
}

\begin{abstract}
Some properties of General Magnetic Fields (GMF) of the Sun as a star and of two solar-like stars (an active young $\xi$ Boo A with no cyclicity of activity, and 61 Cyg A - an old star with a moderate level of cyclic activity) are represented.
\end{abstract}

\section{General Magnetic Field}

The study of large-scale magnetic fields allows us to reveal the main processes causing the activity of a convective star as a whole. GMF is a surface-averaged value of the longitudinal component of magnetic structures. Solar and star' rotation produces observable, periodic variations of this field. The peak value of the GMF of the Sun as a Star (GMFSS) $(\sim 2 \mathrm{G})$ is observed during spot activity maximum (Fig.1A - squares, 1991) and minimal value of the GMFSS $(\sim 0.2 \mathrm{G})$ is observed during spot activity minimum (Fig.1A - open squares, 1986). With the formal examination, such behavior in time demonstrates, to first order, beating of two closely spaced frequencies (see Fig.1 in Kotov et al. 1998). Using periods (Haneychuk et al. 2003) $P_{1}=26 .{ }^{d} 929 \pm 0 .{ }^{d} 015$ and $P_{2}=27 .{ }^{d} 144 \pm 0 .{ }^{d} 015$ we can obtain within errors main prominent periods in the power spectrum for GMFSS and period of activity cycle $\left(9.5^{y} \div 10 .{ }^{y} 5\right)$ (see Table 1 ). One of initial periods is due to a rigid rotation of radiative zone, and the second one is produced by differential rotation. During 35 years of observations, the excess of the positive magnetic flux dominated on the one side of the Sun, and the excess of the negative flux dominated on the opposite side. The GMFSS does not reverse its polarity with the $22 \mathrm{yr}$ solar cycle period. We measure real large-scale field on the Sun with balance of positive and negative magnetic fluxes $\left(\Delta \Phi_{+} / \Delta \Phi_{-} \cong 0.99\right)$ (Plachinda \& Tarasova 2000$)$.

The GMFSS as a phenomenon is absent in the Babcock' and Leighton' phenomenological magneto-kinematic model of the solar cycle. GMFSS is absent in terms of standard $(\alpha-\Omega)$-dynamo theory also. Therefore, the first point of view is "... we measure magnetic disequilibrium of the Sun" (Haneychuk et al. 2003). In this connection, what do we know about GMF on solar-like stars? Here we show results of GMF measurements both for an active young solar-like star $\xi$ Boo A, and for an old solar-like star 61 Cyg A (see Fig.1B and Fig.2A) (Plachinda 2004). In Fig.2B for 61 Cyg A dashed arrows connect progression of the observations in time (with numbering) for process of emergence of Active Regions (ARs). Numerical simulations lead us to the conclusion that in the case of $61 \mathrm{Cyg} \mathrm{A}$ a) more strong magnetic fluxes can be present in ARs $\left(10^{23}-10^{24} \mathrm{Mx}\right)$ than those on the Sun $\left(\sim 5 \times 10^{22} \mathrm{Mx}\right)$; b) ARs can be formed at the same latitudes as on the Sun; c) ARs birth phases may be connected to the "active longitudes". Existence of the GMF on these stars with vigorous convective envelopes confirms a conclusion that the GMF is a real phenomenon and reflects properties of a stationary global magnetic field, Origin Magnetic Field (OMF), of the Sun', convective star', radiative interior onto its surface. OMF appear to be the initial magnetic field for dynamo mechanisms. 
Difference in frequencies

$$
\begin{aligned}
& 1 / 26 .{ }^{d} 93-1 / 27 .{ }^{d} 14 \\
& 2 / 27 .{ }^{d} 14-1 /\left(365 .{ }^{d} 256 \times 9 .{ }^{y} 5\right) \\
& 1 / 26 .^{d} 93+2 \times 1 / 27 .{ }^{d} 14 \\
& 1 / 13 .{ }^{d} 64-1 / 27 .{ }^{d} 14 \\
& 1 / 13 .{ }^{d} 65-1 / 26 .{ }^{d} 91 \\
& 1 / 27 .{ }^{d} 72-2 /\left(10 .^{y} 1 \times 365 .{ }^{d} 256\right)
\end{aligned}
$$

Computed result Actual observation

$\begin{array}{rr}9 .^{y} 5 & \sim 9 .{ }^{y} 5 \div 10 .{ }^{y} 5 \\ 13 .{ }^{d} 62 & 13 .{ }^{d} 62 \\ 9 .{ }^{d} 02 & 9 .^{d} 03 \\ 27 .{ }^{d} 42 & 27 .{ }^{d} 42 \\ 27 .{ }^{d} 70 & 27 .{ }^{d} 72 \\ 28 .{ }^{d} 14 & 28 .{ }^{d} 14\end{array}$

Table 1. Actual and computed periods for GMF of the Sun as a star
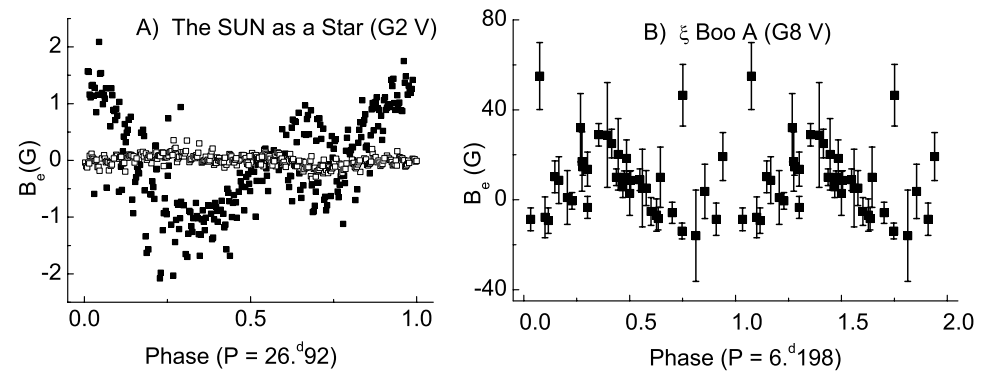

Figure 1 .
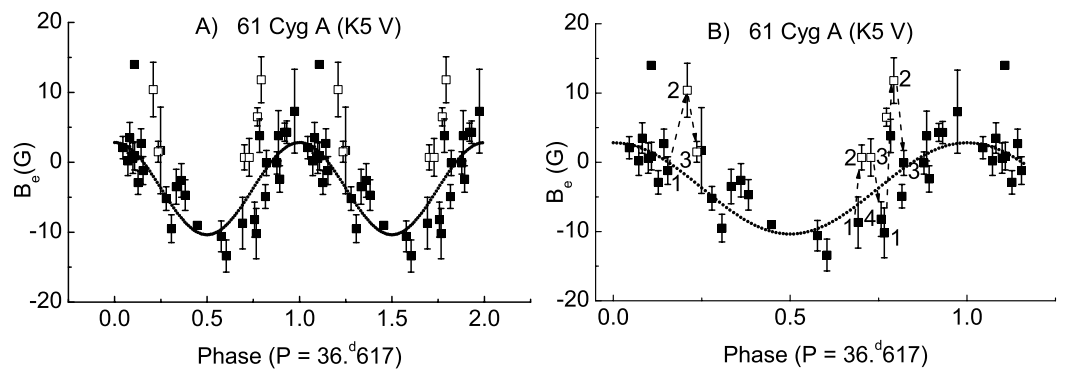

Figure 2.

\section{Acknowledgements}

I thank R. Gershberg, V. Haneychuk, V. Kotov, and T. Tsap for useful discussions. This work has been supported in part by the Ukrainian SFFD grant No. 02.07/00300.

\section{References}

Haneychuk, V. I., Kotov, V. A., \& Tsap, T. T. 2003, A\&A 403, 1115-1121

Kotov, V. A., Scherrer, P. H., Howard, R. F., \& Haneychuk, V. I. 1998, ApJS 116, 103-117. Plachinda, S. I. 2004, in Photopolarimetry in Remote Sensing (eds Videen, G., Yatskiv, Ya. S., \& Mishchenko, M. I.). NATO Science Series Book, Kluwer Acad. Publ., in press.

Plachinda, S. I., \& Tarasova, T. N. 2000, ApJ 533, 1016-1022 